\title{
CDKL5-Related Disorders: From Clinical Description to Molecular Genetics
}

\author{
N. Bahi-Buisson ${ }^{a-c}$ T. Bienvenu ${ }^{a, b, d}$ \\ ${ }^{a}$ Inserm, ${ }^{b}$ Institut Cochin, Université Paris Descartes, ' Neurologie Pédiatrique, Hôpital Necker - Enfants Malades, \\ d Laboratoire de Biochimie et Génétique Moléculaire, Hôpital Cochin, Assistance Publique - Hôpitaux de Paris, \\ Paris, France
}

\section{Key Words}

CDKL5 • Epileptic encephalopathy • Rett syndrome

\begin{abstract}
Mutations in the cyclin-dependent kinase-like 5 gene (CDKL5) have been described in girls with Rett-like features and early-onset epileptic encephalopathy including infantile spasms. To date, with more than 80 reported cases, the phenotype of CDKL5-related encephalopathy is better defined. The main features consist of early-onset seizures starting before 5 months of age, severe mental retardation with absent speech and Rett-like features such as hand stereotypies and deceleration of head growth. On the other hand, neuro-vegetative signs and developmental regression are rare in CDKL5 mutation patients. The CDKL5 gene encodes a serine threonine kinase protein which is characterized by a catalytic domain and a long C-terminal extension involved in the regulation of the catalytic activity of CDKL5 and in the sub-nuclear localization of the protein. To our knowledge, more than 70 different point mutations have been described including missense mutations within the catalytic domain, nonsense mutations causing the premature termination of the protein distributed in the entire open reading frame, splice variants, and frameshift mutations. Additionally, CDKL5 mutations have recently been described in 7 males with a more severe epileptic encephalopathy and a worse
\end{abstract}

outcome compared to female patients. Finally, about 23 male and female patients have been identified with gross rearrangements encompassing all or part of the CDKL5 gene, with a phenotype reminiscent of CDKL5-related encephalopathy combined with dysmorphic features. Even if recent data clearly indicate that CDKL5 plays an important role in brain function, the protein remains largely uncharacterized. Phenotype-genotype correlation is additionally hampered by the relatively small number of patients described.

Copyright $\odot 2011$ S. Karger AG, Base

\section{History of the Disease}

Rett syndrome (RTT; OMIM 312750) is a severe Xlinked neurodevelopmental disorder with a wide spectrum of clinical manifestations. Beside the classic RTT form, several RTT variants have been described, including the early-seizure variant (seizure onset before regression). The early seizure variant was initially described by Hanefeld in 1985, who reported a girl with infantile spasms and hypsarrhythmia in early life [Hanefeld, 1985; Rajaei et al., 2011]. This Hanefeld variant of RTT presents a phenotypic overlap with West syndrome, also known as infantile spasm syndrome, X-linked (ISSX; OMIM 308350). ISSX is characterized by the triad of infantile spasms, hypsarrhythmia, and severe to profound mental

\section{KARGER}

Fax +4161306 1234

E-Mail karger@karger.ch

www.karger.com
(C) 2011 S. Karger AG, Basel

$1661-8769 / 11 / 0025-0137 \$ 38.00 / 0$

Accessible online at:

www.karger.com/msy
Thierry Bienvenu

Institut Cochin

Université Paris Descartes

24 rue du Faubourg St Jacques, FR-75014 Paris (France)

Tel. +33144412479, E-Mail thierry.bienvenu@inserm.fr 
Table 1. Clinical features of female patients with CDKL5 mutations compared to males

\begin{tabular}{lcc}
\hline Clinical features & Female patients/total $(\mathrm{n}=88)$ & Male patients/total $(\mathrm{n}=7)$ \\
\hline Mean age in years at last evaluation (range) & $15(0.6-41)$ & $6(3.5-16)$ \\
Deceleration of head growth & $61 / 72(84.7)$ & $3 / 4(75)$ \\
Regression & $16 / 69(23.2)$ & $3 / 7(42.8)$ \\
Severe intellectual disability & $83 / 85(96.6)$ & $7 / 7(100)$ \\
Poor eye fixation and pursuit & $61 / 72(84.7)$ & $7 / 7(100)$ \\
Language & $9 / 73(12.3)$ & $0 / 7(0)$ \\
Walk with aid or unaided & $24 / 74(32.4)$ & $0 / 7(0)$ \\
Limited hand skills & $47 / 62(75.8)$ & $7 / 7(100)$ \\
Hand stereotypies & $63 / 69(91.3)$ & $1 / 7(14.2)$ \\
Autonomic features & $21 / 50(42)$ & $1 / 7(14.2)$ \\
Early seizures & $86 / 87(98.8)$ & $7 / 7(100)$ \\
Average seizure onset, weeks & $12.5 \pm 9.3$ & $10.9 \pm 9.8$ \\
Infantile spasms/epileptic encephalopathy & $56 / 71(78.8)$ & $7 / 7(100)$ \\
Persistent epilepsy & $47 / 66(71.2)$ & $6 / 7(85.7)$ \\
\hline
\end{tabular}

Patients who presented with a disrupted CDKL5 gene due to unbalanced translocations were excluded. Figures in parentheses are percentages, except where otherwise indicated.

retardation. In 2003, Kalscheuer et al. characterized 2 unrelated female patients with an apparently balanced translocation, 46,X,t $(\mathrm{X} ; 7)(\mathrm{p} 22.3 ; \mathrm{p} 15)$ in 1 case and $46, \mathrm{X}, \mathrm{t}(\mathrm{X} ; 6)(\mathrm{p} 22.3 ; \mathrm{q} 14)$ in the other [Kalscheuer et al., 2003]. The 2 patients presented with a similar phenotype, comprised of severe early-onset infantile spasms with hypsarrythmia and profound global developmental arrest. In both patients, the X-chromosomal breakpoints disrupted CDKL5. In view of the phenotypic overlap between the Hanefeld variant and ISSX, 2 independent groups (in 2004) looked for CDKL5 mutations in patients who had been diagnosed with RTT or a variant of RTT and in whom no MECP2 mutation had been identified. They identified point mutations (such as c.183delT; IVS13-1G>A; p.C152F; p.R175S) in the CDKL5 gene in a subset of patients with a clinical picture resembling the early-onset RTT variant or with a history of early-onset seizures [Tao et al., 2004; Weaving et al., 2004]. It was interesting to note that the seizures seen in the affected individuals reported in these studies and those mentioned in other published reports of CDKL5 defects [Huopaniemi et al., 2000] were generally difficult to control with anticonvulsant therapies. The CDKL5 gene was subsequently analyzed in patients with both classic and atypical variants of RTT by several groups, but mutations were identified only in patients with seizure onset before 6 months of age [Evans et al., 2005; Scala et al., 2005; Archer et al., 2006].

\section{Clinical Features}

CDKL5-related disorders have been mainly reported in females, but 7 affected males with CDKL5 point mutations have been described [Van Esch et al., 2007; Elia et al., 2008; Fichou et al., 2009; Sartori et al., 2009].

\section{Common and Rare Features}

Combining our personal data and the literature, we have listed the most consistent features observed in more than 80 published cases with CDKL5 mutations: (i) normal prenatal history; (ii) irritability, drowsiness and poor sucking in the perinatal period before seizure onset; (iii) earlyonset epilepsy usually before 5 months of age; (iv) Rett-like features consisting of deceleration of head growth, stereotypies, poor to absent voluntary hand use, and sleep disturbances, and (v) severe mental retardation with poor eye contact and virtually no language [Hagberg and Skjeldal, 1994; Bahi-Buisson et al., 2008a; Neul et al., 2010]. Infantile spasms and refractory myoclonic epilepsy are frequent during the course of epilepsy but not always present in CDKL5 mutation patients (tables 1 and 2) [Tao et al., 2004; Weaving et al., 2004; Evans et al., 2005; Mari et al., 2005; Scala et al., 2005; Archer et al., 2006; Buoni et al., 2006; Nectoux et al., 2006; Grosso et al., 2007; Bahi-Buisson et al., 2008b; Pintaudi et al., 2008; Nemos et al., 2009; Russo et al., 2009; Sprovieri et al., 2009; Bahi-Buisson et al., 2010; Mei et al., 2010; Psoni et al., 2010; Arts, 2011; Castren et al., 2011; Melani et al., 2011; Rademacher et al., 2011]. 


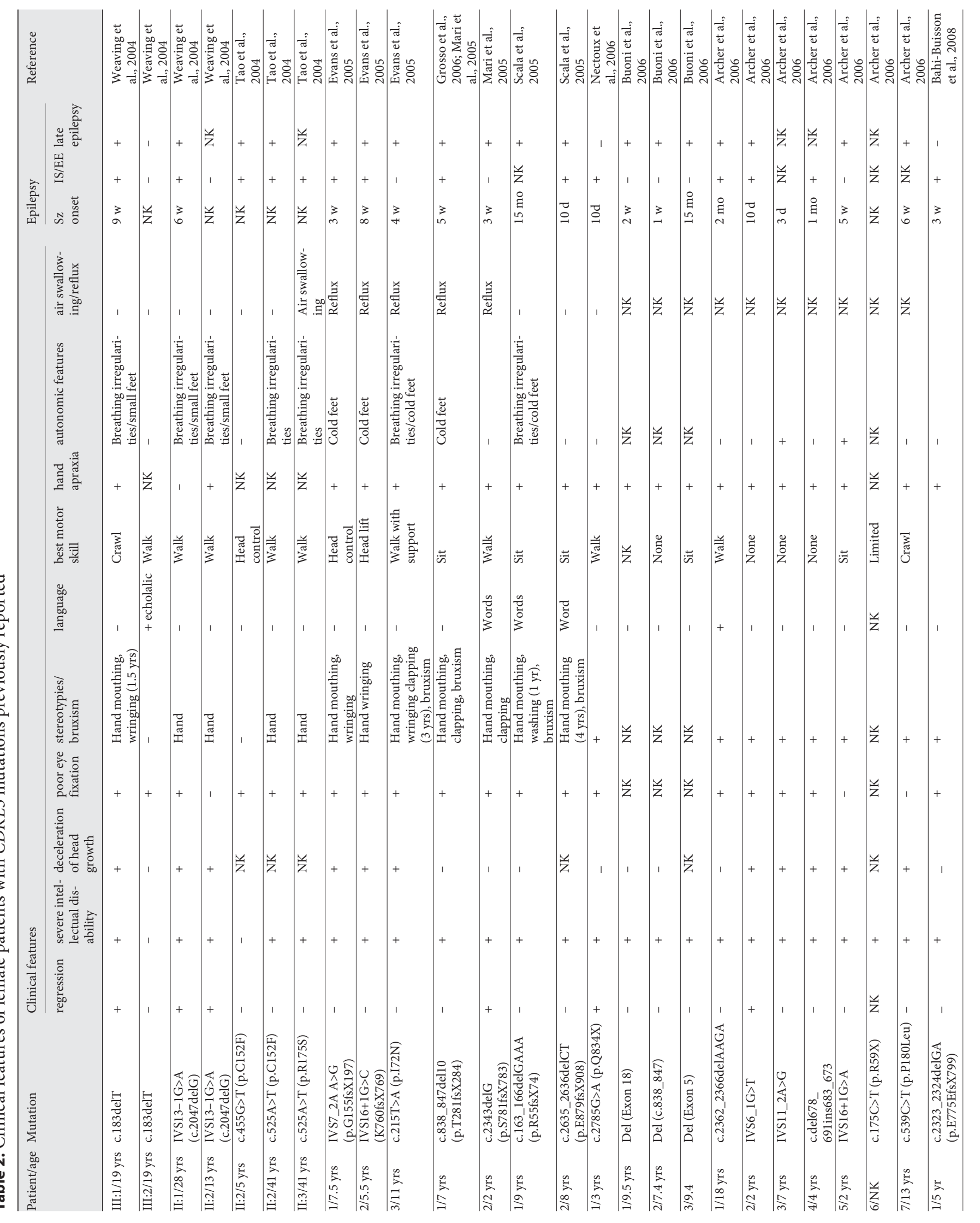


๕ั๊

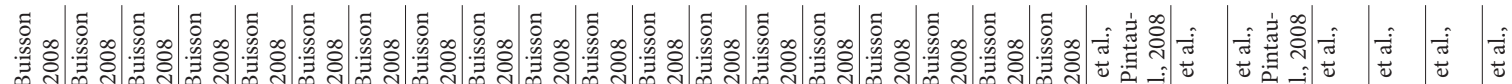

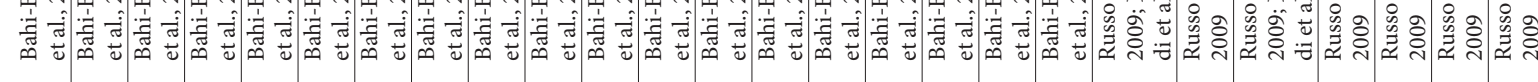

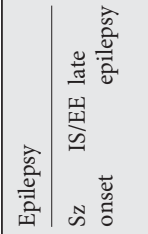

要

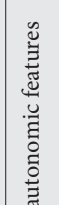

咅

高

彭鿷

美

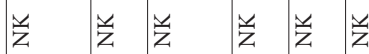

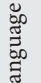

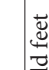

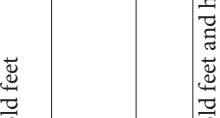

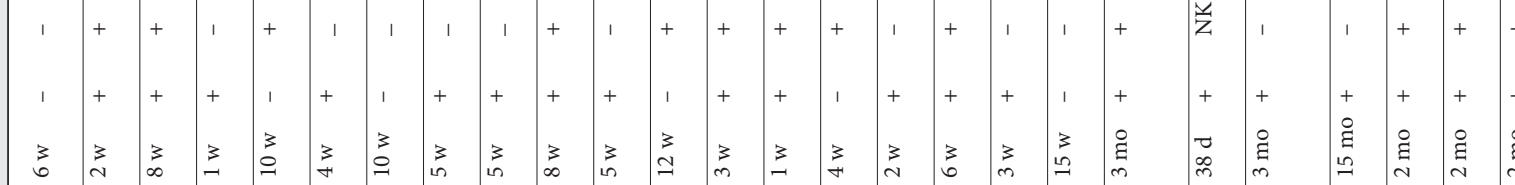

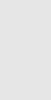

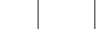
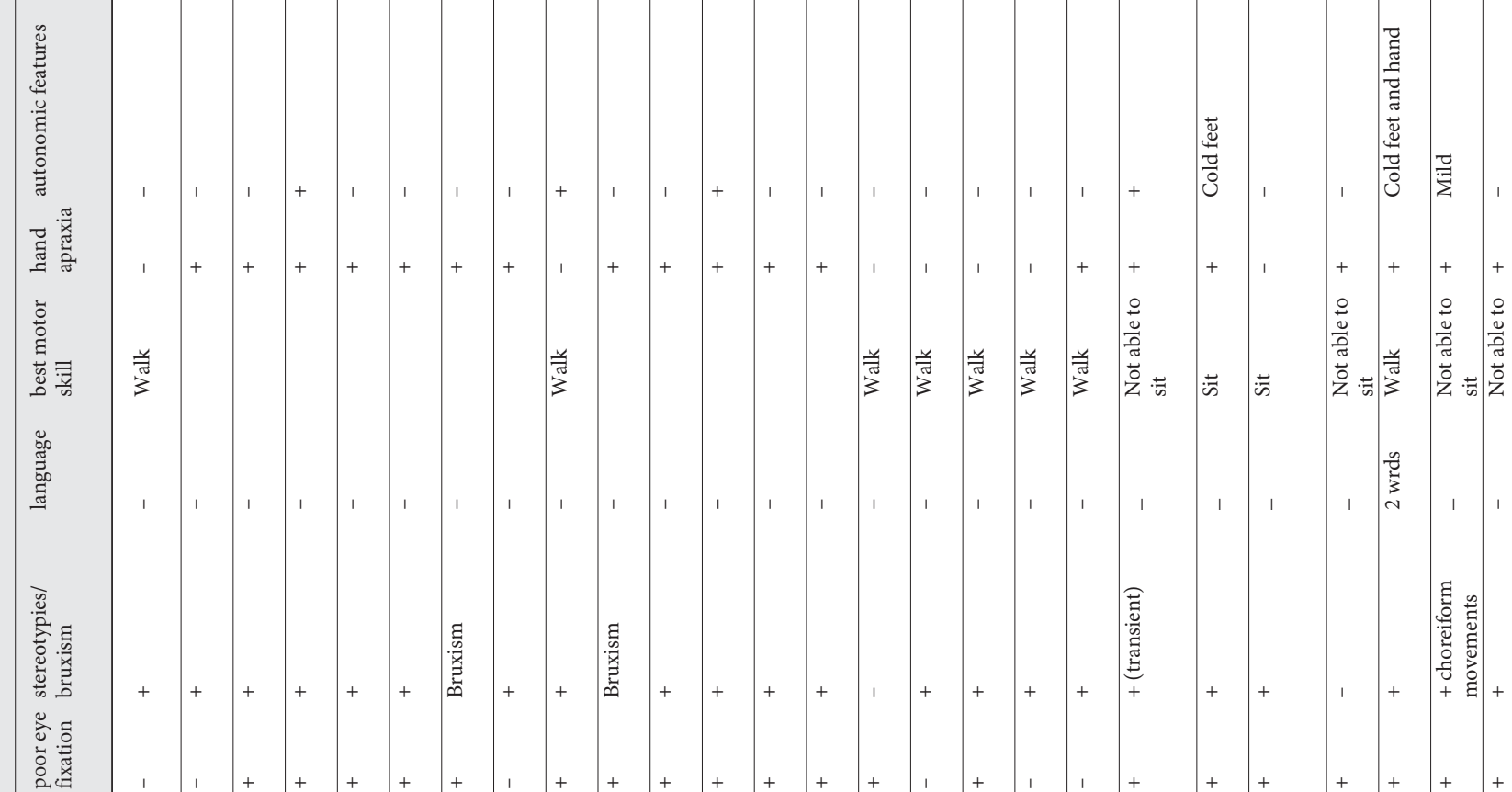

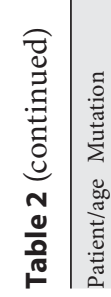

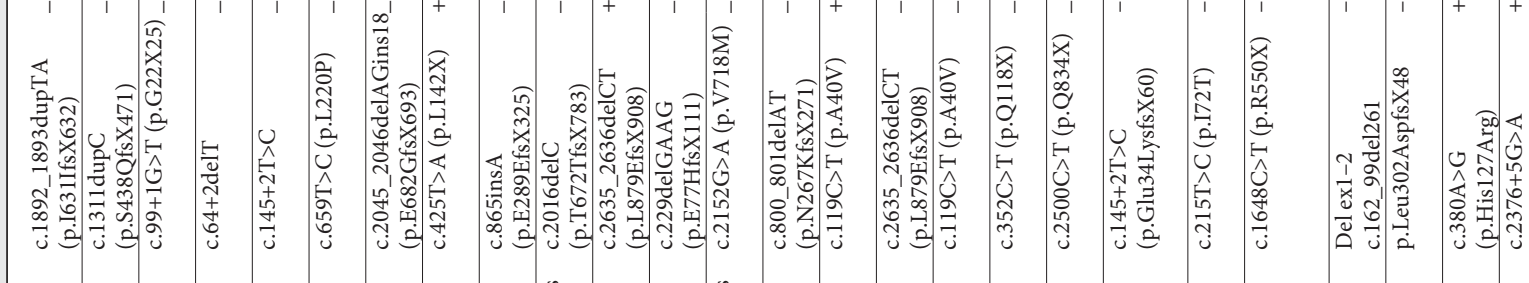

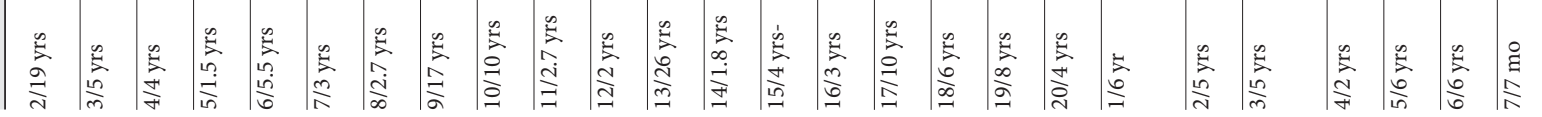




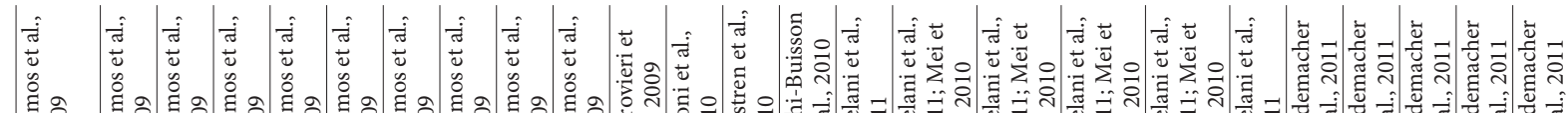

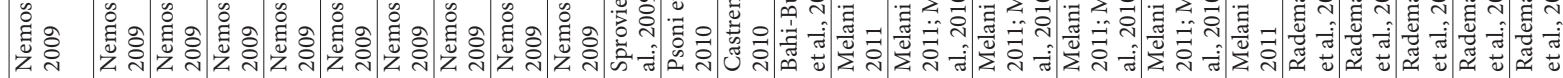

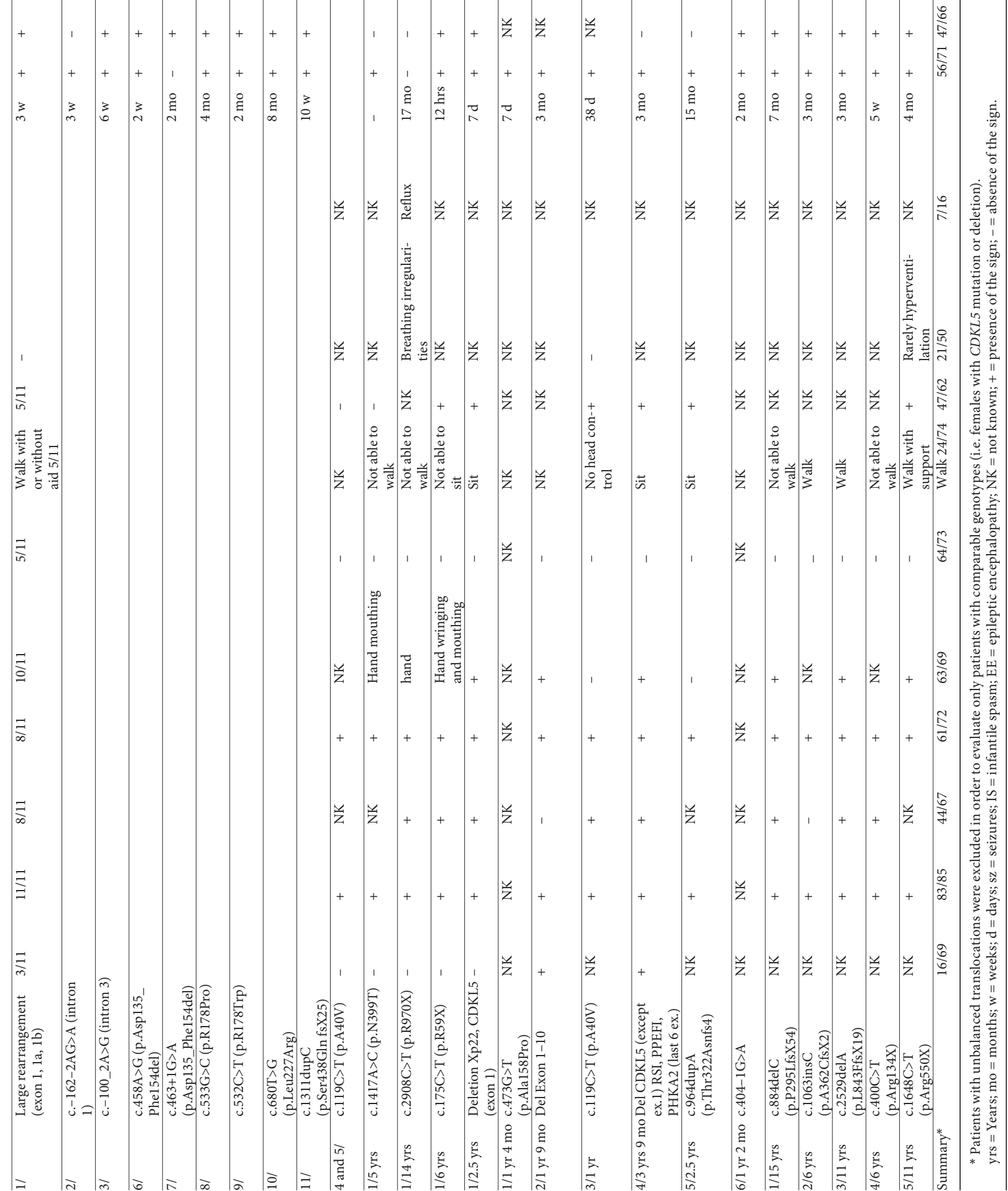


Epileptic encephalopathy is the core symptom of CDKL5-related disorders [Buoni et al., 2006; Bahi-Buisson et al., 2008b; Arts, 2011; Melani et al., 2011]. Seminal descriptions have reported various epileptic phenotypes including infantile spasms [Kalscheuer et al., 2003] and a form of myoclonic encephalopathy [Buoni et al., 2006]. Subsequently, the spectrum of epilepsy has been refined. Our group demonstrated that CDKL5-related epileptic encephalopathy is characterized by a 3-stage evolution consisting of early epilepsy (stage 1), then infantile spasms (stage 2) and, finally, multifocal and refractory myoclonic epilepsy (stage 3) [Bahi-Buisson et al., 2008b].

The epilepsy in stage 1 consists of repetitive prolonged generalized tonic-clonic seizures, with normal or subnormal interictal EEG [Bahi-Buisson et al., 2008b]. This finding is the most frequent in CDKL5 mutation patients. However, Melani et al. demonstrated recently that different degrees of EEG background impairment can be observed, from normal to moderately slow background activity (4/6) with focal or multifocal discharges and, in some cases, highly abnormal EEG with a suppression burst pattern [Melani et al., 2011]. Stage 2 is characterized by infantile spasms and atypical hypsarrhythmia. This pattern occurs in about $75 \%$ of CDKL5 mutation patients. Finally, in stage 3 , the seizures become multifocal and refractory to treatment ( $71 \%$ of patients). The electroclinical features in stage 3 consist of tonic seizures and massive or focal myoclonias on a characteristic background of diffuse highvoltage sharpwaves of 6-7 Hz, and with pseudoperiodic bursts of high-amplitude spikes, polyspikes and spike and waves [Buoni et al., 2006; Bahi-Buisson et al., 2008b].

The large majority of CDKL5 mutation patients show hand stereotypies (91\%). Most patients demonstrate a combination of different hand stereotypies, the most frequent being hand mouthing and clapping. Hand wringing is less frequent. These movements usually appear in the first year of life and become more evident over time [Bahi-Buisson et al., 2008a].

Head circumference is usually within the normal range, or shows a deceleration pattern without absolute microcephaly (85\%). This clearly differs from RTT-related disorders associated with $M E C P 2$ mutations, which is associated with a secondary microcephaly or FOXG1 mutations, which is chararacterized by congenital microcephaly [Bahi-Buisson et al., 2010].

Psychomotor development is severely impaired. Remarkably, the perinatal period is apparently normal; however, irritability, excessive crying and drowsiness are commonly reported from the first weeks of life. About a third of CDKL 5 mutation patients are able to walk. There are phenotypic variations associated with the acquisition of independent ambulation. Those who do not acquire ambulation $(68 \%)$ usually have more severe microcephaly, hand apraxia, poverty of eye gaze, hand use, bruxism and sleep disturbances. Conversely, patients who are able to walk (32\%) have a better eye gaze, hand use, and less bruxism and sleep disturbances. However, the prevalence of refractory epilepsy and hand stereotypies is comparable between the 2 groups [Bahi-Buisson et al., 2008a].

Reduced fetal movements and tactile hypersensitivity, such as dislike of hair brushing, have been reported in a few patients. In addition, some subtle dysmorphic features were noted in 5 patients from the same series. These included deep-set eyes, straight eyebrows, slightly short upturned nose, relatively large ears with large earlobes and high forehead [Archer et al., 2006].

Conversely, some features are rarely observed. Rettlike neurovegetative symptoms such as breathing disturbances, cold extremities and gastrointestinal disturbances are not common. Finally, childhood-onset scoliosis and osteoporosis are uncommon in CDKL5 mutation patients.

Altogether, in CDKL5-related disorders, epileptic seizures are more severe and the neurodevelopmental outcome is worse than in patients with classic Rett syndrome. Interestingly, this impairment tends to predominate on language and hand use. About a third of patients are able to walk, while only a few show purposeful hand use and develop some language. Classic Rett syndrome and CDKL5-related encephalopathy share similar and specific behavioral and neurological traits also called Rett-like features (i.e. deceleration of head growth, hand stereotypies, poor to absent voluntary hand use, and sleep disturbances and virtually no language) that can be useful for the diagnosis.

Brain MRI demonstrates nonspecific abnormalities in CDKL5 mutation patients. Most of them exhibit cortical atrophy combined with hyperintensities in the temporal lobe white matter (fig. 1) [Bahi-Buisson et al., 2008a].

The 7 male patients described had an even more severe encephalopathy and virtually no acquisition of motor skills compared with the CDKL5 mutation females. However, there are some common features, including earlyonset seizures before 3 months, secondary epileptic encephalopathy with infantile spasms and evolution to refractory and myoclonic epilepsy, and severe neurological impairment with lack of visual contact. Of interest, Rettlike features have not been described in males with CDKL5 point mutations [Weaving et al., 2004; Elia et al., 2008; Fichou et al., 2009; Sartori et al., 2009; Castren et al., 2011] (table 3). 

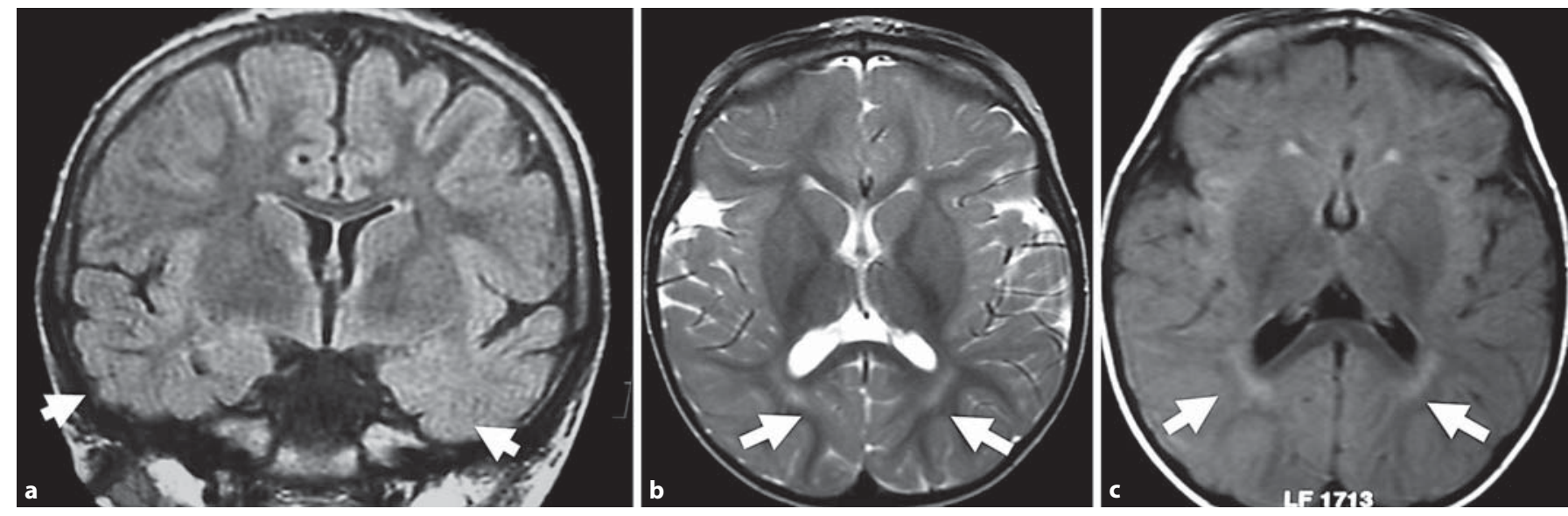

Fig. 1. Brain MRI findings for patients with CDKL5 point mutations. a Nonspecific hyperintensities in both temporal lobes (T2 FLAIR coronal section) in a 12-year-old CDKL5 mutation patient. b Nonspecific periventricular hyperintensities (T2 axial section) in a 3-year-old CDKL5 mutation patient. c Nonspecific periventricular hyperintensities (T2 FLAIR axial section) in a 9-month-old CDKL5 mutation patient. Arrows show the location of the main abnormalities in each figure.

Distinct Features That May Give a Clue to the

Diagnosis in the Light of the Differential Diagnosis

This chapter focuses on the clinical criteria that are of practical value when considering a diagnosis of CDKL5related encephalopathy. A key feature is early-onset epilepsy, usually between the first week and 5 months of age following an unremarkable perinatal period. The hallmark of the disease is the presence of stereotypic hand movements typical of RTT, such as hand-mouthing, hand washing or clapping which become evident after the first year of life. Additional clinical characteristics include severe hypotonia, heavily impaired psychomotor development and normal somatic growth. Other manifestations include poor eye contact and absence of response to social interactions, absence of speech, absence of hand skills and rarer neuro-vegetative dysfunctions such as gastrointestinal disturbances and breathing irregularities [Artuso et al., 2010]. However, CDKL5 mutations have also been identified in a few patients who fulfilled the criteria for a diagnosis of Angelman or Angelman-like syndrome [Russo et al., 2009].

\section{Structure and Functions of the CDKL5 Gene}

\section{The CDKL5 Gene}

CDKL5 (GenBank accession number Y15057) was cloned via an exon trapping method utilized to screen candidate genes in $\mathrm{Xp} 22$, a region where a number of genetic disorders have been found [Montini et al., 1998]. Initially, CDKL5 was found to be composed of 23 exons. The first 3 exons $(1,1 \mathrm{a}$, and $1 \mathrm{~b})$ are untranslated and probably represent 2 transcription start sites of the CDKL5 gene [Kalscheuer et al., 2003]. The initiation codon is located within exon 2. Recently, Fichou et al. reported the identification of an additional 123-bp exon between exons 16 and 17 of the CDKL5 gene, referred to as CDKL5 exon 16b [Fichou et al., 2011] or CDKL5 exon 16a [Rademacher et al., 2011]. This sequence shows a remarkably high degree of similarity between species, suggesting a functional role that has been maintained through evolution. Very interestingly, transcript analysis revealed that the exon 16b-containing mRNA isoform is specifically found in the brain [Fichou et al., 2011]. In rats, 2 splicing isoforms named CDKL5a and CDKL5b (GenBank accession numbers: FJ807484 and GU351881) corresponding to predicted proteins of 934 amino acids and 877 aa in length have also been identified. CDKL5a appears to be the major isoform in pure neuronal cultures, whereas CDKL5b is the only form present in pure glial cultures suggesting a function in gliogenesis [Chen et al., 2010]. Moreover, by aligning both the human and mouse CDKL5 proteins to the orthologs of other species, expression of another CDKL5 isoform with an alternative C-terminus that terminates in intron 18 was described [Williamson et al., 2011]. 


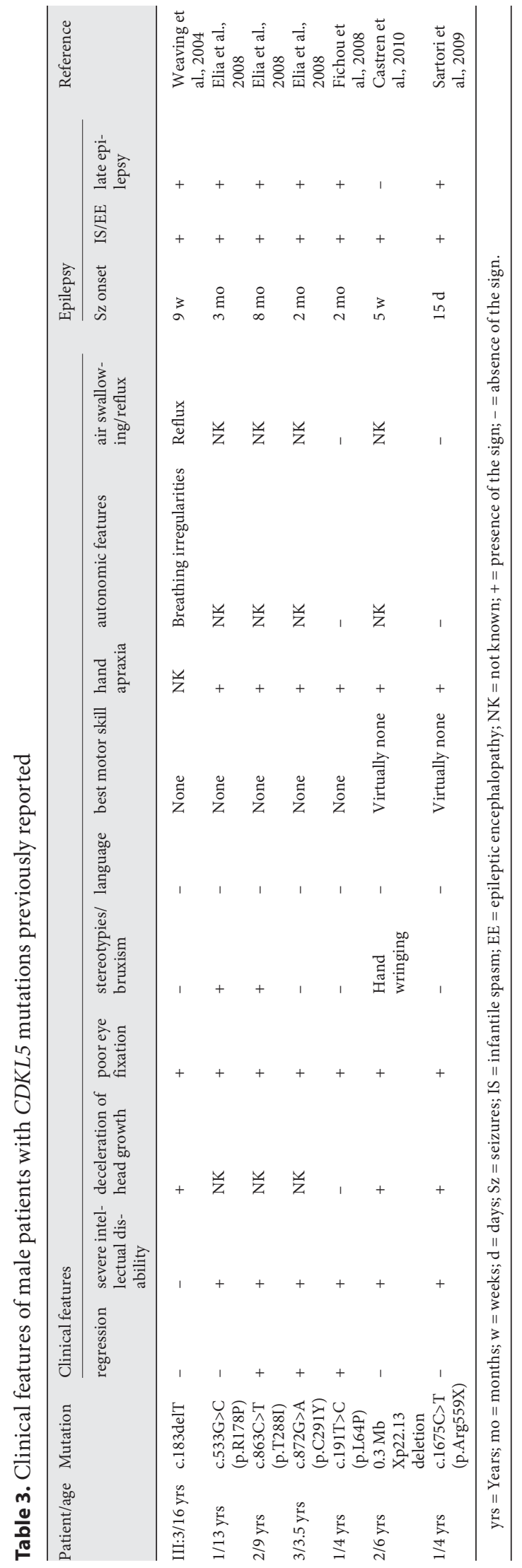

\section{The CDKL5 Protein}

Human CDKL5 is a large protein of 1,030 aa with an estimated molecular mass of $116 \mathrm{kDa}$ [Bertani et al., 2006]. Initially, sequence analysis has indicated that CDKL5 (previously named serine threonine kinase 9, STK9) is a putative proline-directed serine/threonine kinase closely related to p56 KKIAMRE (the protein encoded by CDKL2) and p42 KKIALRE (the protein encoded by CDKL1), which share homology with members of the mitogen-activated protein (MAP) kinase and cyclin-dependent protein kinase-like (CDKL) families [Montini et al., 1998]. The sequence alignment of the CDKL5 protein with these homologs showed 2 kinase signatures in the catalytic domain: an ATP-binding region (amino acids 14-47) and a serine-threonine protein kinase active site (amino acids 127-144) (fig. 2). In addition, CDKL5 has a Thr-Xaa-Tyr sequence that corresponds to the TXY activation motifs of classic MAP kinases such as ERK2, and a putative signal peptidase I serine active site (GTSMCPTL), located between positions 971 and 978 . CDKL5 is also characterized by a remarkably large $\mathrm{COOH}$-terminal extension of almost 700 aa that harbors several functions. Firstly, the tail seems to act as a negative regulator of the catalytic activity of CDKL5, and secondly appears necessary for the nuclear localization of the protein (amino acids 526-780) [Bertani et al., 2006]. However, recently, a new theoretical $107-\mathrm{kDa}$ isoform with an alternative C-terminus that terminates in intron 18 was identified [Williamson et al., 2011], and appeared to be the major isoform in human brain and all other tissues investigated except testis [Williamson et al., 2011].

CDKL5 is a ubiquitous protein that is mainly expressed in the brain (cerebral cortex, hippocampus, cerebellum, striatum, and brain stem), testes and thymus [Lin et al., 2005], shuttling between the nucleus and cytoplasm and varying in different brain areas and during development [Rusconi et al., 2008]. CDKL5 levels appear to be low in the embryonic cortex and are strongly induced at perinatal and postnatal stages in maturing neurons residing in both cerebral cortex and hippocampus. This expression profile is in accordance with the role of the protein in neuronal maturation [Rusconi et al., 2008]. In cultured primary cortical neurons, a significant part of endogenous CDKL5 is present in the nucleus, but a significant fraction is also localized in the dendrites. The subcellular distribution of CDKL5 appears to be modulated through active import and export mechanisms [Rusconi et al., 2008]. The entry of CDKL5 into the nucleus seems to depend on 2 NLS-like stretches (the most 


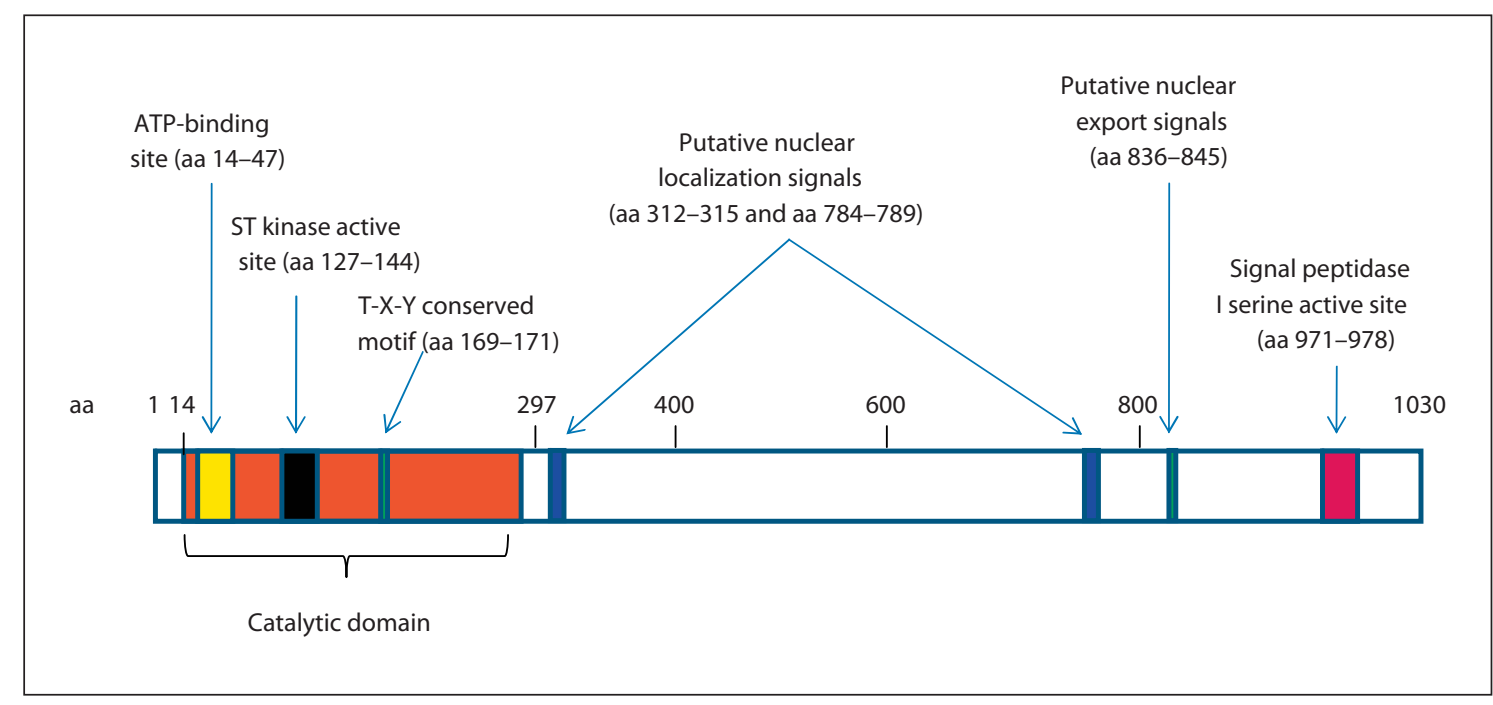

Fig. 2. Schematic illustration of CDKL5. Full-length human CDKL5 is 1,030 aa long and contains the ATPbinding site, the serine-threonine (ST) kinase active site and the conserved Thr-Xaa-Tyr (T-X-Y) motif within the catalytic domain. A putative signal peptidase I serine active site, 2 supposed nuclear localization signals and one supposed nuclear export signal are indicated. The number at the top refers to the amino acid positions.

$\mathrm{N}$-terminal being between amino acids 312 and 315 and the C-terminal between amino acids 784 and 789) of basic amino acids within the long tail of the protein (fig. 2). Moreover, the partial cytoplasmic localization of the fulllength protein depends on nuclear export, mediated by the CRM1 nuclear export receptor [Rusconi et al., 2008].

\section{Functions of the CDKL5 Protein}

CDKL5 is considered a kinase on the basis of sequence homologies. CDKL5 phosphorylation targets are currently largely unknown. However, it has been shown that the protein, in vitro, may autophosphorylate, and it phosphorylates the product of the methyl-CpG-binding protein 2 (MECP2, OMIM 300005) gene, which is mutated in $>90 \%$ of classic RTT patients, suggesting a common signaling pathway between these 2 proteins [Mari et al., 2005]. In addition, CDKL5 specifically interacts with the very $\mathrm{N}$-terminal domain of DNA methyltransferase 1 (DNMT1) in vivo, and phosphorylates significantly DNMT1 in a DNA-dependent manner [Kameshita et al., 2008].

CDKL5 shuttles between the cytoplasm and nucleus through a CRM1-mediated nuclear export mechanism, suggesting a function in both cellular compartments. Recently, Chen et al. studied the effect of CDKL5 knockdown on neurite growth, and found that CDKL5 knockdown resulted in a marked reduction in the total length of both dendrites and axons, suggesting that CDKL5 expression in cortical neurons is required for dendritic arborization. Using in utero electroporated rat brains ablated for CDKL5 they also showed that CDKL5 knockdown delayed neuronal migration as early as $\mathrm{P} 0$ in the rat brain suggesting that migratory defects may be involved in the occurrence of early seizures in patients with CDKL5 mutations. Moreover, they found that CDKL5 regulated neuronal morphogenesis through a mechanism involving BDNF-Racl signaling [Chen et al., 2010]. These data suggest that proper function of CDKL5 requires both kinase activity and correct localization to the cytoplasm, leading to a loss-of-function phenotype similar to that caused by kinase-inactivating mutations.

It is also easy to assume that CDKL5 exerts important nuclear functions, too. Indeed, several studies have demonstrated that CDKL5 acts in the same pathway of $\mathrm{MeCP} 2$. CDKL5 can bind and phosphorylate MeCP2 in vitro and $\mathrm{MeCP} 2$ in turn regulates CDKL5 gene expression, at least in certain brain regions [Mari et al., 2005; Bertani et al., 2006; Carouge et al., 2010]. Further, CDKL5 and DNMT1 colocalize and interact in nuclei. CDKL5 also colocalizes with nuclear speckles involved in the storage and/or modification of pre-mRNA splicing factors and influences alternative splicing in heterologous minigene assays [Ricciardi et al., 2009]. It remains to show whether CDKL5 dysfunction causes a general al- 


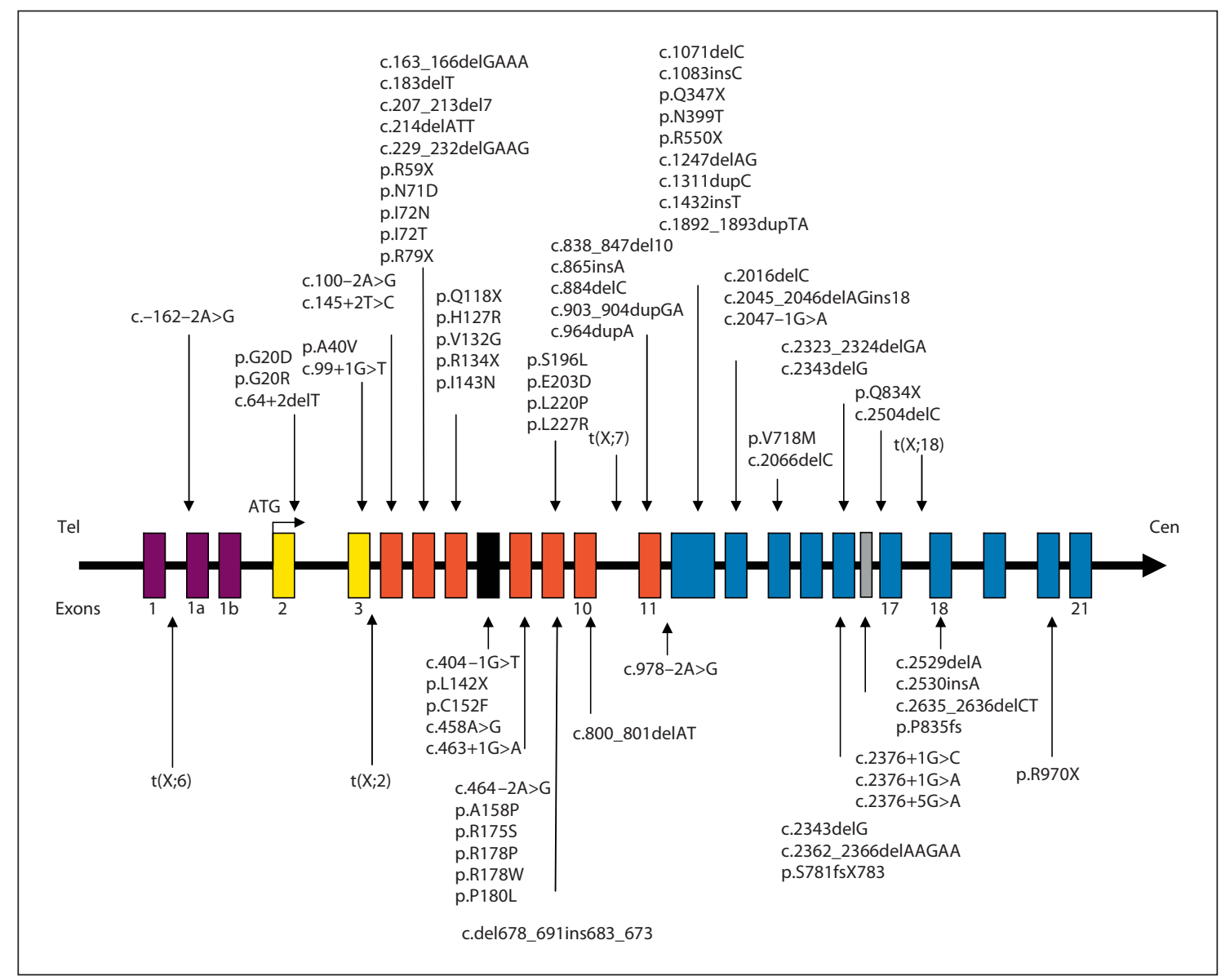

Fig. 3. Schematic representation of the CDKL5 gene. The 3 noncoding exons (1, 1a, and $1 \mathrm{~b})$ are in mauve, exons corresponding to the ATP-binding site are in yellow, and exons corresponding to the serine/threonine kinase active site are in red. The new recently described exon $16 \mathrm{~b}$ is in grey. The positions of reported point mutations, and translocations involving CDKL5 gene identified in female patients are illustrated.

teration in RNA processing and splicing regulation which could be particularly deleterious for the maturation, function and survival of brain neurons.

\section{Mutations in the CDKL5 Gene}

\section{Point Mutations}

Since the first description of point mutations in the CDKL5 gene, more than 70 different point sequence variations have been described resulting in missense, nonsense, splice and frameshift mutations. Twenty-three missense mutations (p.G20R; p.G20D; p.A40V; p.L64P; p.N71D; p.I72N; p.I72T; p.H127R; p.V132G; p.C152F; p.A158P; p.R175S; p.R178W; p.R178P; p.P180L; p.S196L; p.E203D; p.L220P; p.L227R; p.T288I; p.C291Y; p.N399T; p.V718M), 10 nonsense mutations (p.R59X; p.R79X; p.Q118X; p.R134X; p.L142X; p.Q347X; p.R550X; p.R559X;
p.Q834X; p.R970X), 14 splice mutations (c.-162-2A>G; c. $64+2$ delT; $\quad$ c. $99+1 \mathrm{G}>\mathrm{T} ; \quad$ c. $100-2 \mathrm{~A}>\mathrm{G} ; \quad$ c. $145+2 \mathrm{~T}>\mathrm{C}$; c. $404-1 \mathrm{G}>\mathrm{T} ; \quad$ c. $458 \mathrm{~A}>\mathrm{G} ; \quad$ c. $463+1 \mathrm{G}>\mathrm{A} ; \quad$ c. $464-2 \mathrm{~A}>\mathrm{G}$; c. $978-2 A>$ G;c. $2047-1 G>A ; c .2376+1 G>C ; c .2376+1 G>A$; c. $2376+5 \mathrm{G}>\mathrm{A}$ ), and 25 frameshift mutations (c.163 166delGAAA; c.183delT; c.207_213del7; c.229_232delGAAG; c.del678_691ins683_673; c.800_801delAT; c.865insA; c.838_847del10; c.884delC; c.903_904dupGA; c.964dupA; c.1083insC; c.1311dupC; c.1892_1893dupTA; c.2016delC; c.2045_2046delAGins18; c.2066delC; c.2323_ 2324delGA; c.2362_2366delAAGAA; c.2343delG; c.2504delC; c.2529delA; p.S781fsX783; c.2635_2636delCT; p.P835fs) have been described (fig. 3,4$)$. These mutations are spread out all over the coding sequence of CDKL5 with missense mutations almost exclusively found in the kinase domain. As a consequence, missense mutations 


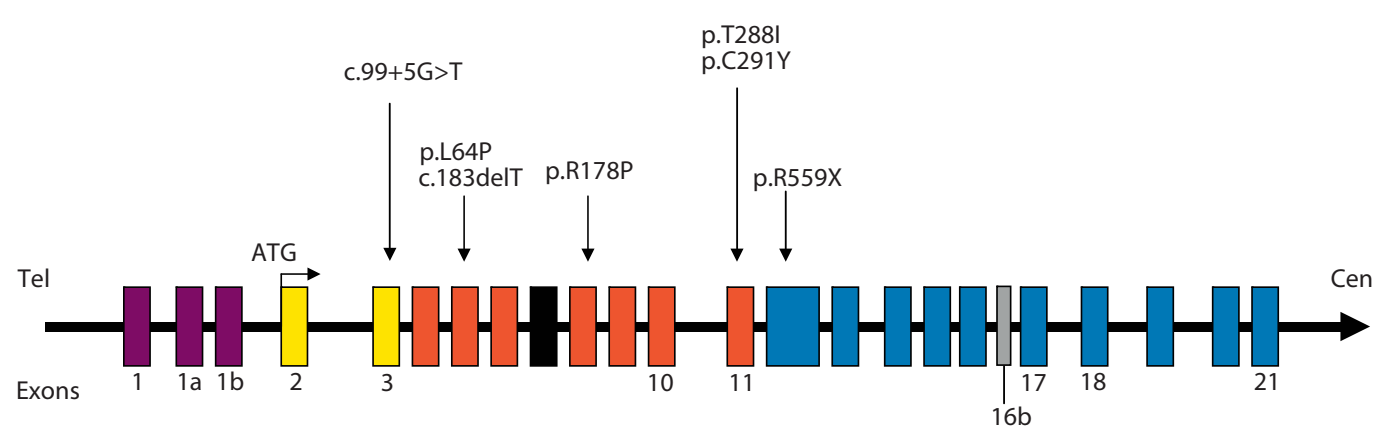

Fig. 4. Gene structure of human CDKL5. The gene consists of 23 exons (21 coding exons 2 to 21). This figure does not include the novel exon 18 including the first 170 nucleotides of intron 18 [Williamson et al., 2011]. The location of the mutations identified in male patients are indicated by arrows at the top. Exons and introns are indicated by open boxes and horizontal lines.

seem to reduce the kinase activity of CDKL5 [Bertani et al., 2006]. Several studies reported impairment in autophosphorylation and phosphorylation of target proteins such as $\mathrm{MeCP} 2$ as a result of missense mutations in the catalytic domain [Tao et al., 2004; Bertani et al., 2006]. In contrast, truncations of the C-terminus seem to enhance its phosphorylation and cause its nuclear accumulation and activity [Rusconi et al., 2008].

Interestingly, recent data suggest that missense mutations negatively affecting the catalytic activity of CDKL5 could be associated with a more severe clinical picture than that caused by truncating mutations located in the last exons of CDKL5 that are predicted to truncate only amino acids of the CDKL5 COOH-terminus [Evans et al., 2005; Bahi-Buisson et al., 2008b; Russo et al., 2009; White et al., 2010].

To date, only 5 recurrent CDKL5 mutations have been reported: c.119C $>\mathrm{T}$ (p.A40V) reported in 5 patients [Bahi-Buisson et al., 2008b; Rosas-Vargas et al., 2008; Nemos et al., 2009], c.533G >C (p.R178W) [Evans et al., 2005; Nemos et al., 2009] and c.2635_2636delCT both reported in 3 unrelated patients each [Elia et al., 2008; Nemos et al., 2009], and c.145+2 T >C [Bahi-Buisson et al., 2008a; Pintaudi et al., 2008] and c.175C > T (p.R59X) both reported in 2 unrelated patients each [Archer et al., 2006; Ricciardi et al., 2009].

The CDKL5 mutations so far reported have almost exclusively been detected in female patients. To date, only 7 males have been reported with point mutations in the CDKL5 gene. The scarcity of known male CDKL5 mutations may be explained, in part, by the fact that far more affected girls than boys have been screened for CDKL5 mutations. Additional studies need to be carried out to better estimate the frequency of CDKL5 mutations in males and females. Strikingly, these male patients show a similar clinical course to females. However, they exhibit more severe neurological features, extreme hypotonia and virtually no psychomotor development in combination with refractory seizures. In 1 boy presenting with a mixed seizure disorder of the 'Lennox-Gastaut' type with profound neurodevelopmental delay, spastic quadriparesis, cortical blindness, and a marked kyphoscoliosis, a frameshift mutation (c.183delT) was identified [Weaving et al., 2004]. In 4 others with severe or profound mental retardation and early-onset intractable seizures, missense mutations (p.L64P; p.R178P; p.T288I; p.C291Y) were identified [Elia et al., 2008; Fichou et al., 2011]. A de novo nonsense mutation p.R559X was identified in a 4 -year-old boy with a 47,XXY karyotype presenting with a severe early-onset epileptic encephalopathy and profound intellectual and motor impairment with features reminiscent of RTT [Sartori et al., 2009]. Finally, we recently reported on a boy displaying a somatic mosaicism for a new splice mutation c. $99+5 \mathrm{G}>\mathrm{A}$ within the CDKL5 gene, providing evidence that somatic mosaicism could explain several cases of severe encephalopathy and early-onset epilepsy in males (fig. 4) [Masliah-Plachon et al., 2010].

\section{Deletions and Duplications}

In 2000, Huopaniemi et al. described a large $136-\mathrm{kb}$ inherited deletion, truncating the 3 '-end of the CDKL5 gene and including both the RS1 and PPEF1 genes, in a 
male with retinoschisis, mild hemiplegia, and epilepsy [Huopaniemi et al., 2000]. Seven years later, Van Esch et al. described a 10 -month-old boy with a de novo $2.8-\mathrm{Mb}$ deletion at Xp22.2-Xp22.13. The clinical phenotype present in this male patient consisted of severe encephalopathy, myoclonic seizures, bilateral congenital cataract and tetralogy of Fallot. The deleted region encompasses 16 genes or transcripts of which most are ubiquitously expressed, including the brain. The only known diseaseassociated genes in this region are the NHS gene (NanceHoran syndrome) and exons 1-11 of the CDKL5 gene [Van Esch et al., 2007]. In the same year, the same group from Leuven identified a large Xpter duplication of $21 \mathrm{Mb}$ including CDKL5 in a 2-year-old boy with severe mental retardation, generalized hypotonia, and facial dysmorphim, nasal hypoplasia, antimongoloid position of the palpebral fissures, and a flat occiput. However, the parental DNA was not analyzed and the de novo nature of this duplication was therefore not proven [Froyen et al., 2007]. In 2008, another large duplication of $8.5 \mathrm{Mb}$ including CDKL5 was found in a man with severe psychomotor development delay [Tzschach et al., 2008]. Since these first reports, several teams have identified variably-sized microdeletions involving several or all exons of the CDKL5 gene in females with early-onset seizures, by cytogenetic analysis and/or by comparative genomic hybridization (CGH) array and/or by multiplex ligation-dependent probe amplification assay (MLPA). Large rearrangements removing only few exons of the CDKL5 gene without affecting the neighboring genes have been reported in 11 girls with early-onset seizures (exons 1-1a-1b [Nemos et al., 2009]; exons 2-3 [Russo et al., 2009]; 0.11-0.19 Mb of exons 1-3 [White et al., 2010]; 57-kb deletion of exons 1-3, $137-\mathrm{kb}$ deletion of exons $1-4,74-\mathrm{kb}$ deletion of exons $1-3$ [Erez et al., 2009]; 4-kb deletion of exons 7-8, 180-kb deletion of exons 1-10 [Mei et al., 2010; Bartnik et al., 2011]. In 2 documented cases, deletions were flanked by Alu repetitive elements and may have resulted from either nonallelic homologous recombination or the microhomology-mediated fork stalling and template switching/microhomology-mediated break-induced replication mechanism [Erez et al., 2009]. Moreover, large deletions involving part or the entire genomic region of CDKL5 and several additional genes (such as RS1, PPEF1, PHKA2, KISS1R, SCML2) have been identified in another 4 female patients [Bahi-Buisson et al., 2010; Mei et al., 2010; Castren et al., 2011]. All these recent data highlight the importance of screening for large intragenic and whole gene deletions.

\section{Genetics of CDKL5 Disorders}

\section{Mode of Inheritance}

The CDKL5-related disorder is an X-chromosomal dominantly inherited disorder. Up to now, all described cases except one [Weaving et al., 2004] are simplex cases (i.e., a single occurrence in a family), resulting from a de novo mutation. Only 1 familial case with 3 affected individuals has been described [Weaving et al., 2004]. Because of the possibility of germline mosaicism, it is appropriate to offer prenatal diagnosis to couples who had a child with a CDKL5-related disorder regardless of whether the disease-causing mutation has been detected in a parent or not.

\section{Frequency of CDKL5 Mutations and Molecular Diagnosis}

The prevalence of these CDKL5-related disorders has not been estimated, but they appear to be rare. The overall frequency of CDKL5 mutations in females with early-onset seizures is around $8-16 \%$ [Bahi-Buisson et al., 2008a; Nemos et al., 2009; Mei et al., 2010]. Of note, the CDKL5 mutation rate is higher (28\%) in female patients with early-onset seizures combined with infantile spasms [BahiBuisson et al., 2008a]. The diagnosis of all CDKL5-related disorders relies on molecular genetic and cytogenetic testing. Sequence analysis and deletion testing for exonic and whole-gene deletions are available on a clinical basis.

Point mutations in the CDKL5 coding region are detected by bidirectional sequencing and/or mutation scanning (e.g. DHPLC). Deletion/duplication analysis is carried out by a variety of methods including real-time quantitative PCR, quantitative multiplex fluorescent PCR, MLPA, and/or CGH-microarray. Once a putative pathogenic mutation has been identified in a proband, it is appropriate to offer testing to all first-degree relatives regardless of their clinical status. If a putatively pathogenic mutation was identified in an unaffected mother, $\mathrm{X}$-chromosome inactivation should be performed in the mother and her daughter. This test will help to distinguish a pathogenic mutation from benign polymorphism.

\section{Genotype-Phenotype Correlation}

The phenotypes associated with CDKL5 mutations range from a milder form where the epilepsy is controlled and independent ambulation is achieved to a severe form with microcephaly, virtually no motor development and refractory epilepsy. The severity is at least associated with the proportion of functional or partially functional CDKL5 protein produced by the normal or mutated al-
Mol Syndromol 2011;2:137-152
Bahi-Buisson/Bienvenu 
leles, respectively, and associated with the variability of $\mathrm{X}$-chromosome inactivation. Given the small number of patients in each series, genotype-phenotype correlation studies have been limited. However, it has been suggested that mutations affecting the $\mathrm{N}$-terminal catalytic domain are associated with a more severe phenotype consisting of earlier onset and intractable infantile spasms followed by late-onset multifocal myoclonic epilepsy [Bahi-Buisson et al., 2008b]. Additionally, Russo et al. observed that patients with stop codon mutations have a milder phenotype than those with missense or splicing mutations [Russo et al., 2009]. A possible explanation would be that some missense mutations can lead to a mislocalization of the CDKL5 mutated protein, caused by amino acid substitution in the nuclear import domains [Rosas-Vargas et al., 2008]. A more extensive knowledge of the currently restricted CDKL5 mutational repertoire might provide new clues concerning phenotype-genotype correlations and analyzing synthetic derivatives of particular mutants in functional assays might highlight new protein domains.

The CDKL5 gene is subject to $\mathrm{X}$-chromosome inactivation (XCI) and it has been proposed that skewed patterns could explain phenotypic variability in females heterozygous for a mutated allele. Consistent with this hypothesis, the most severe phenotype, with early-onset encephalopathy, was present in the females who carried the X;autosome translocations, in which the normal X chromosome was completely inactivated [Kalscheuer et al., 2003]. Variable expression of the wild-type CDKL5 allele has been shown to explain intrafamilial phenotypic variability of the disease [Tao et al., 2004]. However, the twin sisters described by Weaving et al. [2004] exhibited completely different clinical features from each other but had a similar XCI pattern in cells from peripheral blood. Subsequent data also suggested that the majority of CDKL5 mutation patients show a random pattern of XCI in blood leukocytes [Bahi-Buisson et al., 2008a; Nemos et al., 2009]. In accordance with these findings, the clinical heterogeneity in unrelated CDKL5 mutation patients may not only be due to XCI. However, we cannot rule out preferential inactivation of the normal allele in brain tissues in patients with severe phenotype, and that the XCI pattern in blood leukocytes may not reflect the XCI patterns in the brain.

\section{Management and Treatment}

Unfortunately, there are currently no specific treatments that halt or reverse the progression of the CDKL5related disease. As a consequence, the management is mainly symptomatic and aimed at maximizing developmental and cognitive potential. A dynamic multidisciplinary approach is necessary with specialist inputs from dietitians, physiotherapists and occupational therapists.

Epilepsy is one of the main problems in most CDKL5 mutation patients and is largely refractory to medication. Antiepileptic drugs should be used according to the electroclinical pattern. More specifically, antiepileptic drugs like valproate or vigabatrin can be used during stage 1 . For infantile spasms, steroids are recommended, according to the recent recommendations of the infantile spasms working group [Pellock et al., 2010].

When patients develop myoclonic and multifocal epilepsy, most antiepileptic drugs fail to control seizures. Some drugs can be useful such as levetiracetam, for example to treat myoclonic seizures [Vigevano, 2005] or topiramate [French et al., 2004] for tonic seizures. Finally, ketogenic diet therapy can be effective (personal data). Vagus nerve stimulation has been of limited, if any benefit [Evans et al., 2005].

Pharmacological approaches also include the use of melatonin for sleep disturbances. Attention needs to be paid to nutritional problems and the development of spasticity, both of which can have a major impact on quality of life in disabled patients. Psychosocial support for the families is an integral part of the management.

\section{State of Research}

In contrast to $M E C P 2$, a mouse model for CDKL5-related disease is currently lacking, limiting our knowledge of the function of CDKL5 inside the neuron. Although conditional Cdkl5 mouse mutant lines and recombinant Cdkl5 embryonic stem cells have been generated by different groups, up to now, no mutant male mice $\left(\mathrm{Cdkl5}^{-/ \mathrm{y}}\right)$ or heterozygous $\left(\mathrm{Cdkl}^{-/+}\right)$females are available. Recently, however, Chen et al. have used RNA interference to silence CDKL5 expression in rat cortical neurons [Chen et al., 2010]. They demonstrated that CDKL5 is essential for neuronal morphogenesis since its downregulation in cultured neurons inhibits neurite outgrowth and dendritic arborization, while its overexpression elicits the opposite effect through a signaling pathway involving the Rac1 GTPase. Interestingly, CDKL5 deficiency alters the expression of ASK1/MAP3K5, known to be activated by Rac1, in nonneuronal cells [Nectoux et al., 2011]. This suggests that reduction of both ASK1/MAP $3 K 5$ expression and activation in CDKL5-deficient cells contribute to the pathogenesis of CDKL5 deficiency on neuronal 
morphogenesis. To find out if these data recapitulate the situation in affected human brain, several groups will propose to reprogram fibroblasts from patients carrying different CDKL5 mutations into induced pluripotent stem cells with the aim to establish a human neuronal model for CDKL5-associated disease, which will be better suited to understand the disease pathogenesis [Amenduni et al., 2011].

Another fascinating challenge is to find out whether $M E C P 2$ and $C D K L 5$, both associated with Rett syndrome, are involved in a common molecular pathway. At the molecular level, MeCP2 and CDKL5 form a protein complex in vivo and the catalytic activity of CDKL5 mediates directly or indirectly the phosphorylation of $\mathrm{MeCP} 2$ in vitro [Mari et al., 2005; Bertani et al., 2006]. Moreover, the 2 genes share temporal and spatial expression patterns in the brain and are simultaneously activated during neuronal maturation and synaptogenesis [Mari et al., 2005; Rusconi et al., 2008]. According to this model, CDKL5 may work upstream of MeCP2 influencing directly or indirectly its phosphorylation state and thereby specific functions of MeCP2. In the absence of CDKL5, these phosphorylation-dependent activities of MeCP2 would be altered causing a subset of RTT symptoms. However, it remains to be elucidated which residues are phosphorylated by CDKL 5 and how MeCP 2 activity is influenced by these modifications.

CDKL5 shuttles between the cytoplasm and nucleus through a CRM1-mediated nuclear export mechanism. In the nucleus, CDKL5 localizes and is associated with a number of splicing factors that are clustered in structures called 'nuclear speckles'. CDKL5 is involved in the structural organization of nuclear speckles and the dynamics of their components. Surprisingly, CDKL5 ectopic expression is sufficient to induce disassembly of speckles, both in cell lines and primary human fibroblasts. Conversely, its downregulation obtained by means of shRNAmediated silencing technology leads to consistent larger speckles. Accordingly, CDKL5 mutated human primary fibroblasts display abnormal large and uneven speckles [Ricciardi et al., 2009]. Interestingly, the alterations of nuclear speckles caused by CDKL5 misexpression appear mostly dependent on its kinase activity [Ricciardi et al., 2009]. These results suggest that CDKL5 might exert a control on these subnuclear structures by influencing the cycle of phosphorylation and dephosphorylation of their associated SR proteins. The resulting imbalance of the various components of the spliceosome machinery ultimately leads to alteration of the splicing pattern of a number of undefined RNA transcripts. Therefore, it remains to show whether CDKL5 dysfunction causes a general alteration in RNA processing and splicing regulation which could be particularly deleterious for the maturation, function and survival of brain neurons.

Finally, in the cytoplasm, CDKL5 appears to regulate neuronal morphogenesis via BDNF-Racl signaling [Chen et al., 2010]. CDKL5 colocalizes and forms a protein complex with Rac1, a critical regulator of actin remodeling and neuronal morphogenesis. However, it is not clear how CDKL 5 affects the activity of Rac1. Discovering new targets of the CDKL5 kinase would be fascinating to understand the mechanism by which CDKL5 influences neuronal morphogenesis.

\section{Conclusion}

Mutations in the CDKL5 gene have been identified in female patients with early-onset epileptic encephalopathy and severe mental retardation with a RTT-like phenotype [Kalscheuer et al., 2003]. RTT-like features become evident only after the first year of life [Bahi-Buisson et al., 2008a]. Subsequently, CDKL5 mutations have been shown to be associated with more diverse phenotypes including mild epilepsy, autism without epilepsy [Evans et al., 2005; Scala et al., 2005] and an Angelman-like phenotype [Tao et al., 2004]. Up to now, more than 90 mutations have been described including missense mutations within the catalytic domain, nonsense mutations causing the premature termination of the protein distributed in the entire open reading frame, splice variants, frameshift mutations, and large molecular rearrangements involving part or the entire genomic region of CDKL5. Additional CDKL5 mutations have recently been described in males with a more severe epileptic encephalopathy and a worse outcome compared to female patients. CDKL5 is a kinase that autophosphorylates and phosphorylates the product of the MECP 2 gene, the major gene involved in classic RTT, suggesting that CDKL5 acts on the same pathway as MeCP2. On the other hand, CDKL5 has cellular functions not related to $M E C P 2$. Of these, recent data on neurons demonstrates an essential role of CDKL5 on neuronal morphogenesis and neuritic outgrowth, through a pathway involving the Racl GTPase. However, a mouse model for CDKL5-related encephalopathy is still lacking. 


\section{References}

Amenduni M, De Filippis R, Cheung AY, Disciglio V, Epistolato MC, et al: iPS cells to model CDKL5-related disorders. Eur J Hum Genet (2011).

-Archer HL, Evans J, Edwards S, Colley J, Newbury-Ecob R, et al: CDKL5 mutations cause infantile spasms, early onset seizures, and severe mental retardation in female patients. J Med Genet 43:729-734 (2006).

Arts WF: CDKL5 gene-related epileptic encephalopathy: electroclinical findings in the first year of life. Dev Med Child Neurol 53:296297 (2011).

-Artuso R, Mencarelli MA, Polli R, Sartori S, Ariani F, et al: Early-onset seizure variant of Rett syndrome: definition of the clinical diagnostic criteria. Brain Dev 32:17-24 (2010).

-Bahi-Buisson N, Nectoux J, Rosas-Vargas R, Milh M, Boddaert N, et al: Key clinical features to identify girls with CDKL5 mutations. Brain 131:2647-2661 (2008a).

- Bahi-Buisson N, Kaminska A, Boddaert N, Rio $M$, Afenjar A, et al: The three stages of epilepsy in patients with CDKL5 mutations. Epilepsia 49:1027-1037 (2008b).

-Bahi-Buisson N, Girard B, Gautier A, Nectoux J, Fichou Y, et al: Epileptic encephalopathy in a girl with an interstitial deletion of Xp22 comprising promoter and exon 1 of the CDKL5 gene. Am J Med Genet B Neuropsychiatr Genet 153B:202-207 (2010).

- Bartnik M, Derwinska K, Gos M, Obersztyn E, Kolodziejska KE, et al: Early-onset seizures due to mosaic exonic deletions of CDKL5 in a male and two females. Genet Med 13:447452 (2011).

-Bertani I, Rusconi L, Bolognese F, Forlani G, Conca B, et al: Functional consequences of mutations in CDKL5, an X-linked gene involved in infantile spasms and mental retardation. J Biol Chem 281:32048-32056 (2006).

Buoni S, Zannolli R, Colamaria V, Macucci F, di Bartolo RM, et al: Myoclonic encephalopathy in the CDKL5 gene mutation. Clin Neurophysiol 117:223-227 (2006).

- Carouge D, Host L, Aunis D, Zwiller J, Anglard $\mathrm{P}: C D K L 5$ is a brain $\mathrm{MeCP} 2$ target gene regulated by DNA methylation. Neurobiol Dis 38: 414-424 (2010).

-Castren M, Gaily E, Tengström C, Lähdetie J, Archer $\mathrm{H}$, et al: Epilepsy caused by CDKL $5 \mathrm{mu}-$ tations. Eur J Paediatr Neurol 15:65-69 (2011).

-Chen Q, Zhu YC, Yu J, Miao S, Zheng J, et al: CDKL5, a protein associated with Rett syndrome, regulates neuronal morphogenesis via Racl signaling. J Neurosci 30:1277712786 (2010).

- Elia M, Falco M, Ferri R, Spalletta A, Bottitta M, et al: CDKL5 mutations in boys with severe encephalopathy and early-onset intractable epilepsy. Neurology 71:997-999 (2008).
Erez A, Patel AJ, Wang X, Xia Z, Bhatt SS, et al: Alu-specific microhomology-mediated deletions in CDKL5 in females with early-onset seizure disorder. Neurogenetics 10:363-369 (2009).

Evans JC, Archer HL, Colley JP, Ravn K, Nielsen $J B$, et al: Early onset seizures and Rett-like features associated with mutations in CDKL5. Eur J Hum Genet 13:1113-1120 (2005).

Fichou Y, Bieth E, Bahi-Buisson N, Nectoux J, Girard B, et al: Re: CDKL5 mutations in boys with severe encephalopathy and early-onset intractable epilepsy. Neurology 73:77-78; author reply 78 (2009).

Fichou Y, Nectoux J, Bahi-Buisson N, Chelly J, Bienvenu T: An isoform of the severe encephalopathy-related CDKL5 gene, including a novel exon with extremely high sequence conservation, is specifically expressed in brain. J Hum Genet 56:52-57 (2011).

-French A, Kanner AM, Bautista J, Abou-Khalil B, Browne T, et al: Efficacy and tolerability of the new antiepileptic drugs II: treatment of refractory epilepsy. Report of the Therapeutics and Technology Assessment Subcommittee and Quality Standards Subcommittee of the American Academy of Neurology and the American Epilepsy Society. Neurology 62:1261-1273 (2004).

Froyen G, Bauters M, Boyle J, Van Esch H, Govaerts K, et al: Loss of SLC38A5 and FTSJ1 at Xp11.23 in three brothers with non-syndromic mental retardation due to a microdeletion in an unstable genomic region. Hum Genet 121:539-547 (2007).

-Grosso S, Brogna A, Bazzotti S, Renieri A, Morgese G, et al: Seizures and electroencephalographic findings in CDKL5 mutations: case report and review. Brain Dev 29: 239-242 (2007).

Hagberg BA, Skjeldal OH: Rett variants: a suggested model for inclusion criteria. Pediatr Neurol 11:5-11 (1994).

Hanefeld F: The clinical pattern of the Rett syndrome. Brain Dev 7:320-325 (1985).

-Huopaniemi L, Tyynismaa H, Rantala A, Rosenberg T, Alitalo T: Characterization of two unusual RS1 gene deletions segregating in Danish retinoschisis families. Hum Mutat 16: 307-314 (2000).

Kalscheuer VM, Tao J, Donnelly A, Hollway G, Schwinger E, et al: Disruption of the serine/ threonine kinase 9 gene causes severe Xlinked infantile spasms and mental retardation. Am J Hum Genet 72:1401-1411 (2003).

Kameshita I, Sekiguchi M, Hamasaki D, Sugiyama Y, Hatano N, et al: Cyclin-dependent kinase-like 5 binds and phosphorylates DNA methyltransferase 1. Biochem Biophys Res Commun 377:1162-1167 (2008).

Lin C, Franco B, Rosner MR: CDKL5/Stk9 kinase inactivation is associated with neuronal developmental disorders. Hum Mol Genet 14:3775-3786 (2005).
Mari F, Azimonti S, Bertani I, Bolognese F, Colombo E, et al: CDKL5 belongs to the same molecular pathway of MeCP2 and it is responsible for the early-onset seizure variant of Rett syndrome. Hum Mol Genet 14:19351946 (2005).

Masliah-Plachon J, Auvin S, Nectoux J, Fichou Y, Chelly J, et al: Somatic mosaicism for a CDKL5 mutation as an epileptic encephalopathy in males. Am J Med Genet A 152A:2110 2111 (2010).

Mei D, Marini C, Novara F, Bernardina BD, Granata T, et al: Xp22.3 genomic deletions involving the $C D K L 5$ gene in girls with early onset epileptic encephalopathy. Epilepsia 51: 647-654 (2010).

-Melani F, Mei D, Pisano T, Savasta S, Franzoni E, et al: CDKL5 gene-related epileptic encephalopathy: electroclinical findings in the first year of life. Dev Med Child Neurol 53:354360 (2011).

Montini E, Andolfi G, Caruso A, Buchner G, Walpole SM, et al: Identification and characterization of a novel serine-threonine kinase gene from the Xp22 region. Genomics 51: 427-433 (1998).

Nectoux J, Heron D, Tallot M, Chelly J, Bienvenu $\mathrm{T}$ : Maternal origin of a novel C-terminal truncation mutation in CDKL5 causing a severe atypical form of Rett syndrome. Clin Genet 70:29-33 (2006).

-Nectoux J, Fichou Y, Cagnard N, Bahi-Buisson $\mathrm{N}$, Nusbaum P, et al: Cell cloning-based transcriptome analysis in cyclin-dependent kinase-like 5 mutation patients with severe epileptic encephalopathy. J Mol Med 89:193202 (2011).

Nemos C, Lambert L, Giuliano F, Doray B, Roubertie A, et al: Mutational spectrum of CDKL5 in early-onset encephalopathies: a study of a large collection of French patients and review of the literature. Clin Genet 76: 357-371 (2009).

-Neul JL, Kaufmann WE, Glaze DG, Christodoulou J, Clarke AJ, et al: Rett syndrome: revised diagnostic criteria and nomenclature. Ann Neurol 68:944-950 (2010).

- Pellock JM, Hrachovy R, Shinnar S, Baram TZ, Bettis D, et al: Infantile spasms: a U.S. consensus report. Epilepsia 51:2175-2189 (2010).

Pintaudi M, Baglietto MG, Gaggero R, Parodi E, Pessagno A, et al: Clinical and electroencephalographic features in patients with CDKL 5 mutations: two new Italian cases and review of the literature. Epilepsy Behav 12: 326-331 (2008).

Psoni S, Willems PJ, Kanavakis E, Mavrou A, Frissyra H, et al: A novel p.Arg970X mutation in the last exon of the CDKL5 gene resulting in late-onset seizure disorder. Eur J Paediatr Neurol 14:188-191 (2010). 
Rademacher N, Hambrock M, Fischer U, Moser $B$, Ceulemans B, et al: Identification of a novel CDKL5 exon and pathogenic mutations in patients with severe mental retardation, early-onset seizures and Rett-like features. Neurogenetics 12:165-167 (2011).

-Rajaei S, Erlandson A, Kyllerman M, Albage M, Lundstrom I, et al: Early infantile onset 'congenital' Rett syndrome variants: Swedish experience through four decades and mutation analysis. J Child Neurol 26:65-71 (2011).

-Ricciardi S, Kilstrup-Nielsen C, Bienvenu T, Jacquette A, Landsberger N, et al: CDKL5 influences RNA splicing activity by its association to the nuclear speckle molecular machinery. Hum Mol Genet 18:4590-4602 (2009).

-Rosas-Vargas H, Bahi-Buisson N, Philippe C, Nectoux J, Girard B, et al: Impairment of CDKL5 nuclear localisation as a cause for severe infantile encephalopathy. J Med Genet 45:172-178 (2008).

Rusconi L, Salvatoni L, Giudici L, Bertani I, Kilstrup-Nielsen C, et al: CDKL5 expression is modulated during neuronal development and its subcellular distribution is tightly regulated by the C-terminal tail. J Biol Chem 283:30101-30111 (2008).
Russo S, Marchi M, Cogliati F, Bonati MT, Pintaudi $\mathrm{M}$, et al: Novel mutations in the CDKL5 gene, predicted effects and associated phenotypes. Neurogenetics 10:241-250 (2009).

-Sartori S, Di Rosa G, Polli R, Bettella E, Tricomi $\mathrm{G}$, et al: A novel CDKL5 mutation in a $47, \mathrm{XXY}$ boy with the early-onset seizure variant of Rett syndrome. Am J Med Genet A 149A:232-236 (2009).

Scala E, Ariani F, Mari F, Caselli R, Pescucci C, et al: CDKL5/STK9 is mutated in Rett syndrome variant with infantile spasms. J Med Genet 42:103-107 (2005).

Sprovieri T, Conforti FL, Fiumara A, Mazzei R, Ungaro C, et al: A novel mutation in the $\mathrm{X}$-linked cyclin-dependent kinase-like 5 (CDKL5) gene associated with a severe Rett phenotype. Am J Med Genet A 149A:722725 (2009).

Tao J, Van Esch H, Hagedorn-Greiwe M, Hoffmann K, Moser B, et al: Mutations in the X-linked cyclin-dependent kinase-like 5 (CDKL5/STK9) gene are associated with severe neurodevelopmental retardation. Am J Hum Genet 75:1149-1154 (2004).

Tzschach A, Chen W, Erdogan F, Hoeller A, Ropers $\mathrm{HH}$, et al: Characterization of interstitial Xp duplications in two families by tiling path array CGH. Am J Med Genet A 146A:197-203 (2008).
-Van Esch H, Jansen A, Bauters M, Froyen G, Fryns JP: Encephalopathy and bilateral cataract in a boy with an interstitial deletion of Xp22 comprising the CDKL5 and NHS genes. Am J Med Genet A 143:364-369 (2007).

Vigevano F: Levetiracetam in pediatrics. J Child Neurol 20:87-93 (2005).

Weaving LS, Christodoulou J, Williamson SL, Friend KL, McKenzie OL, et al: Mutations of CDKL5 cause a severe neurodevelopmental disorder with infantile spasms and mental retardation. Am J Hum Genet 75:1079-1093 (2004).

White R, Ho G, Schmidt S, Scheffer IE, Fischer $A$, et al: Cyclin-dependent kinase-like 5 (CDKL5) mutation screening in Rett syndrome and related disorders. Twin Res Hum Genet 13:168-178 (2010).

Williamson SL, Giudici L, Kilstrup-Nielsen C, Gold W, Pelka GJ, et al: A novel transcript of cyclin-dependent kinase-like 5 (CDKL5) has an alternative $\mathrm{C}$-terminus and is the predominant transcript in brain. Hum Genet (2011). 